\title{
Dynamique sociale des techniques biomédicales
}

\section{Jean Martin}

Ancien médecin cantonal vaudois et membre de la Commission nationale d'éthique

\section{«Emancipation civile» des avancées scientifiques}

Par ce néologisme, j'aimerais discuter le fait que les progrès de la sphère biomédicale développent dans la communauté une vie propre, prenant de plus en plus de place, avec une sorte d'autonomie. Même si le plus souvent - et c'est bien ainsi - l'évolution des mœurs précède celle de la loi, les choses semblent s'accentuer aujourd'hui, au plan de l'intensité du phénomène comme de sa rapidité. Qu'on pense à ce que nous avons vécu depuis 1978 pour la procréation médicalement assistée: la naissance de Louise Brown a suscité quantité d'interrogations et de réserves qui, aujourd'hui et s'agissant des méthodes principales de PMA, ont pratiquement disparu (à propos des enjeux du registre psychologique et de l'imaginaire - voire ontologique - dans ce domaine, à noter l'ouvrage récent de François Ansermet, Marc Germond et de trois historiennes de l'art [1]. Cette dynamique des progrès techniques, nous la voyons aussi en matière de recherche, de transplantation, de clonage (animal ... à ce stade), et certainement de génétique - cf. la multiplication des usages des tests ADN.

Nombre de pratiques se sont instaurées avant que des cadres n'aient été posés (entre autres par le législateur). Tout en étant attaché comme je le suis à une société libérale, où tout n'a pas à être entériné par les pouvoirs publics, il y a néanmoins matière à réflexion. Il est souvent souhaité, quant au principe, que des instances d'éthique adéquatement mandatées, et selon les cas les pouvoirs publics, prennent position sur tels moyens nouveaux avant leur application à une certaine échelle voire de routine. En réalité, les usages s'installent sans que les conséquences «civiles», au sens large, en aient été examinées de manière approfondie. D'où le terme d'émancipation suggéré plus haut.

\section{Des normes établies informellement et plus par des mécanismes institutionnels - un exemple}

Dans son numéro du 15 décembre 2007, l'hebdomadaire français Marianne publie un article de Christian Godin [2] sur les applications de la génétique, en rapport avec l'amendement législatif
Mariani prévoyant l'utilisation de tests ADN pour vérifier l'appartenance familiale lors de regroupement de personnes immigrées (voir [3]). Godin écrit: «La légalité et la moralité caractérisaient l'ordre ancien dont la modernité sait faire l'économie. Et point n'est besoin que la norme soit imposée pour qu'elle s'impose. Dans les pays de l'Europe du Nord, on assiste depuis quelques années à une chute brutale du nombre des naissances d'enfants malformés; le diagnostic préimplantatoire permet en effet l'élimination de fœtus présentant des anomalies génétiques. Il y a là un eugénisme civil, non étatique, qui n'a été recommandé par aucun gouvernement. Seulement, un Etat qui aurait pris des décisions aboutissant au même résultat eût été immanquablement qualifié de nazi» (c'est nous qui soulignons).

Et plus loin: «La norme qui s'impose est en effet plus contraignante que celle que l'on impose. L'homme moderne s'est laissé peu à peu enfermer dans une prison douce et invisible, la plus dorée de toutes: la prison de la santé. La génétique en ses débuts avait un usage médical et judiciaire [...]. Dans l'ombre, de manière diffuse, un usage économique l'emporte de plus en plus sur le politique.» Ici: par usage économique, il faut entendre la mise en œuvre de moyens sans qu'il y ait eu évaluation du point de vue de la res publica, de la Cité; par «le politique», il faut entendre ce qui est décidé sur la place publique, notamment les cadres légaux votés par les parlements.

Prééminence de l'économique, de la consommation que certains appelleront «démocratique»: choisissant leurs habitudes, les consommateurs votent avec leurs pieds et leur porte-monnaie; il est vrai qu'on ne peut nier qu'il y ait demande de techniques nouvelles. Chosification souvent ... Godin encore: «La biologisation de la société va de pair avec la marchandisation de l'existence.»

\section{Eugénisme civil, familial, domestique?}

Restons à l'exemple du diagnostic préimplantatoire (DPI) afin d'éviter la gestation d'enfants porteurs de troubles graves. Sociétalement, eugénisme est un «mot du Mal». Il y a le propos fou des Nazis, il y a toutes les formes de purification ethnique. Mais, dans notre profession (chez les gynécologues, pédiatres, généticiens ...) et si on 
considère les directions intensément poursuivies de la recherche, beaucoup concourt à améliorer la qualité (merci de ne pas prendre ombrage du mot) des enfants qui naissent et des jeunes et adultes qu'ils deviendront: promouvoir leur «qualité» intrinsèque afin d'augmenter leur qualité de vie. Il y a là des démarches typiques de ce que Godin dénomme eugénisme civil; qu'on peut dire aussi familial voire domestique - home eugenics -, en référence à son caractère privé dans le cadre du colloque singulier, sous le couvert du secret médical. «Eugenik von unten» ... [4]. C'est indéniablement une dimension de la médecine actuelle.

\section{Dans le futur: risques de rejet des personnes présentant des handicaps?} Comme elle le fait depuis toujours en s'efforçant d'améliorer la santé et le «fonctionnement» des individus, la médecine s'engage donc en vue de limiter le nombre de ceux présentant des handicaps. Depuis une vingtaine d'années, j'ai été amené à prendre conscience des réserves émises par les milieux de personnes handicapées ou de leurs proches à l'endroit d'effets possibles de ces avancées: à mesure que ces personnes deviendront plus exceptionnelles, «différentes», le souci est qu'elles susciteront plus de surprise, d'inconfort voire de rejet de la part d'autres moins habitués à les rencontrer, à communiquer et interagir avec elles. Une telle conséquence n'étant certainement pas exclue, la préoccupation est légitime, fondée sur des valeurs de nondiscrimination que nous partageons tous.

Cela étant, quelque chose de raisonnable peut-il/devrait-il être entrepris en rapport avec les méthodes biomédicales qui ont pour effet de diminuer la proportion de personnes physiologiquement et anatomiquement différentes parmi nous? Qui freinera les scientifiques, et les parents/familles qui les sollicitent pour que naissent des enfants disposant des meilleures chances de départ? Est-il envisageable de définir des limites entre les affections qu'il est légitime de chercher à prévenir, voire à éradiquer, et celles qui ne devraient pas l'être (pas du tout, ou selon les cas)?* L'idée serait inepte de suggérer que telle commission d'experts - aussi sophistiqués ces derniers soient-ils - pourrait proposer une proportion optimale de handicapés dans une société donnée et à un moment donné? On retrouve ici la constante difficulté, en bioéthique, à fixer des limites, des «barrières», dans la pratique comme dans la recherche [5]. Malgré la considération que méritent les craintes évoquées, on ne voit donc guère comment formuler une objection de fond à l'usage de techniques visant à éviter la concrétisation de handicaps, ni quelles démarches du domaine de la pratique seraient susceptibles de faire disparaître ces craintes.

Ce qui est possible par contre et pour quoi le corps médical parmi d'autres doit s'engager, c'est une exigence de continuer à sensibiliser et éduquer - chacun et tout le monde - à une convivialité sereine et cordiale avec les personnes différentes. Cela vaut pour celles présentant des troubles de nature physiologique, «médicale», et cela vaut aussi, il est bon de le rappeler, pour celles qui sont différentes psychologiquement, socialement, par leur origine, leur langue, ou d'autres caractéristiques encore.

\section{«Pentes glissantes»}

Les évolutions évoquées ici alimentent la crainte de la pente glissante (slippery slope). Citation à ce sujet: «L'argument de la pente glissante n'est pas dépourvu de pertinence psychologique et sociologique: il rappelle utilement les faiblesses de la volonté et de la raison humaines [...]. Transféré du domaine de la morale de tous les jours à celui de l'évaluation des évolutions des sciences, cet argument devient toutefois d'un maniement difficile et souvent contestable [...]. Cette conception est foncièrement sceptique quant à la capacité des individus de juger et de décider rationnellement et librement dès lors qu'ils auraient fait un premier choix qui, via divers amalgames, pourrait conduire à des entreprises apparentées mais immorales.» [6]

Pas question ici de proposer une appréciation définitive entre l'insistance sur un principe de précaution strictement observé et la confiance faite à l'homme et à sa capacité de juger - capacité qui est au cœur de toute éthique. Il est bon que restent vifs les débats sur ce point entre ceux qu'on peut dire - sommairement - les optimistes d'un côté, les pessimistes de l'autre.

\section{En guise de conclusion - vivre avec I'ambivalence}

«Le biopouvoir ne s'exerce pas seulement par la domination despotique, il opère sur fond de négation du conflit. Dans l'Etat moderne, le conflit n'existe pas, il n'y a plus que des problèmes techniques auxquels des mesures techniques apporteront des solutions techniques.» [7] Nos sociétés vivent de nombreux conflits mais, effectivement, tendent à les nier: un exemple en est l'influence démesurée du politiquement correct. Et un sentiment que la technique résoudra nos soucis est certainement présent - manifeste ou sous-jacent. Ce n'est plus la croyance au progrès d'il y a un siècle, catégorique voire naïve; c'est plutôt une sorte de myopie confortable qui nous fait nous 
reposer sur les potentialités de la technique, sans donner assez d'attention à ce qu'elles entraînent avec elles en termes de formatage voire de contrôle social. Face à cela: comme on réintroduit dans les Hautes Ecoles les humanités (humanities) dans les études scientifiques, ne serait-il pas important qu'elles reprennent une place suffisante, accrue, dans la vie de la Cité?

$\mathrm{Au}$ reste, les choses ne sont jamais simples. Un ami sociologue à qui je soumets ce texte (et qui vient de passer par une transplantation hépatique), me fait les commentaires suivants: «De plus en plus, il faut apprendre à vivre avec l'ambivalence, nous devons exister avec des choses qui ne sont pas résolues par la seule raison, qui nous dépassent ... Il y a des prix à payer pour disposer/bénéficier du progrès, prix qui dépend entre autres de la culture et de l'époque; il est vrai que certaines demandes faites à la technologie sont absurdes et, dans la mesure du possible, il convient de les marginaliser. De plus, toujours il y a eu des aspects mythiques dans l'approche de la santé et de la maladie (l'épilepsie vue comme (mal sacrés) et il n'est pas étonnant que, sous diverses formes, le mythe accompagne les avancées actuelles. C'est aussi le cas pour les transplantations, sujet avec lequel je viens de me colleter intensément. Il ne faut pas croire que l'ambivalence est essentiellement une marque de faiblesse, elle peut être un moment nécessaire de l'approche du «mieux possible et de la gestion d'un mystère constitutif de l'existence.» De l'existence personnelle et sociétale!

Gérer l'ambivalence, c'est le besoin depuis Aristote de rechercher les justes milieux, en pondérant les circonstances, aspirations, droits et intérêts en cause. C'est aussi se rapprocher de l'objectif pratique de l'éthique: comment faire pour bien faire?

\section{Références}

1 Ansermet F, Germond M, Mauron V, André M, Cascino F. Clinique de la procréation et mystère de l'incarnation - L'ombre du futur. Paris: Presses Universitaires de France; 2007.

2 Godin C. La biologisation de la société est en marche. Marianne. 2007;(556):74-6.

3 Martin J. Tests génétiques chez les personnes venues d'ailleurs. Bull Méd Suisses. 2007;88(47): 2022.

4 Taverna E. Human Enhancement - Füllhorn oder Büchse der Pandora? Bull Méd Suisses. 2008; 89(4):143-4.

5 Martin J. Quo vadis, éthique en biomédecine? Rev Méd Suisse. 2007;3:1973-4.

6 Hottois G. Pente glissante. Dans: Hottois G, Missa J-N. Nouvelle Encyclopédie de Bioéthique. Bruxelles: De Boeck Université; 2001, p. 640-1 (voir aussi dans le même ouvrage: Godard O. Principe de précaution, p. 650-8).

7 Benasayag M, del Rey A. Eloge du conflit. Paris: La Découverte; 2007.

8 Enhancement-Medizin. SGBEbulletin. 2008; (56).

* NB: est évoquée ici la prévention de troubles altérant significativement les chances de développement et la qualité de vie des (futures) personnes concernées; je n'aborde pas le «enhancement», les pratiques dites amélioratives, qui à l'évidence posent question, et suis opposé à l'idée d'une - hypothétique - routinisation de la PMA pour permettre de choisir les caractéristiques de l'enfant à naître - sauf une fois encore quand il s'agit d'écarter une caractéristique pathologique sérieuse. Ceci tout étant péniblement conscient de ce que rapporte Taverna [4] à propos d'un colloque (novembre 2007) de l'Académie Suisse des Sciences Médicales consacré au «human enhancement» (notre traduction): «Plusieurs exposés et discussions ont bientôt montré que ni les méthodes thérapeutiques ni celles de prévention, telles qu'on les comprend jusqu'ici, ne se distinguent clairement des nouvelles techniques amélioratives possibles. Parce que les conceptions de la maladie suivent des normes sociétales et que la recherche nous confronte toujours plus vite à des connaissances partielles nouvelles [...].» Lors de la même réunion, le Prof. Stella Reiter-Theil, de l’Université de Bâle, a relevé que ce débat soulève inévitablement la question du principe bioéthique de non-malfaisance. Elle estime que si l'enhancement ne donne lieu à aucun bénéfice de nature thérapeutique, il convient d'être particulièrement prudent/restrictif. Et, au-delà des aspects individuels, il faut examiner les effets/risques sociaux au cas où ces méthodes ne seraient réservées ou accessibles qu'à un petit nombre. De plus, le médecin ne saurait assumer à lui seul le «filtrage», les choix à faire. Une discussion interdisciplinaire au sein de la société s'impose. La question est d'importance majeure: qui dira dans l'avenir ce qu'il est admissible d'améliorer et ce qui ne l'est pas? (Voir le Bulletin de mars 2008 de la Société suisse pour l'éthique biomédicale, consacré aux exposés donnés dans le cadre du colloque susmentionné de l'ASSM [8].) 